\title{
Cholesteric Mesophase in Aqueous Solutions of a Triple Helical Polysaccharide Scleroglucan
}

\author{
Toshio Yanaki,* Takashi Norisuye, and Akio Teramoto \\ Department of Macromolecular Science, Osaka University, \\ Toyonaka, Osaka 560, Japan \\ (Received November 16, 1983)
}

\begin{abstract}
Concentrated aqueous solutions of two samples of triple helical scleroglucan with molecular weights $1.93 \times 10^{5}$ and $1.24 \times 10^{5}$ were studied by polarizing microscopy and optical rotatory dispersion at temperatures from $11-35^{\circ} \mathrm{C}$. As the polymer weight fraction $w$ was increased, birefringent phases appeared in the continuous isotropic phase at $w \sim 0.14$ and 0.19 (for the higher- and lower-molecular weight samples, respectively) and spread over the entire region of the solution at $w \sim 0.19$ and 0.31 , almost regardless of temperature. The microscopic patterns of these birefringent phases resembled those reported for cholesteric liquid crystals. The specific rotation $[\alpha]_{436}$ at $21.5^{\circ} \mathrm{C}$ markedly increased with increasing $w$ between 0.14 and 0.19 and leveled off at a value about 700 times the infinite dilution value. From these findings, it was concluded that the birefringent phase of aqueous scleroglucan is cholesteric. The intrinsic birefringence of the scleroglucan triple helix in the cholesteric solution was estimated from the optical rotation data to be $(1.5 \pm 0.5) \times 10^{-2}$

KEY WORDS Polysaccharide / Scleroglucan / Triple Helix / Cholesteric Liquid Crystal / Phase Equilibrium / Optical Rotatory Dispersion / Birefringence /
\end{abstract}

Polytran scleroglucan is a capsular polysaccharide elaborated by the fungus, Sclerotium rolfsii. As shown in our previous papers, ${ }^{1,2}$ this polysaccharide consists essentially of the same repeating units as those of schizophyllan, an extracellular $\beta$-1,3-D-glucan produced by Schizophyllum commune, and disperses in pure water or dilute aqueous sodium hydroxide as a rodlike triple helix very similar to that of schizophyllan, ${ }^{3-5}$ provided the molecular weight of the sample in aqueous solution is lower than about $6 \times 10^{5}$.

Recently, Van et al. ${ }^{6}$ found that schizophyllan in water forms a cholesteric mesophase at a polymer concentration above about $10 \%$. From this fact along with the abovementioned similarities between scleroglucan and schizophyllan, we expected that aqueous scleroglucan would also form a cholesteric liquid crystalline phase when the concentration was above a certain value.

In this work, we prepared two samples of Polytran scleroglucan and investigated their aqueous solutions ranging in concentration from zero to about $35 \%$, by polarizing microscopy and optical rotatory dispersion (ORD).

\section{EXPERIMENTAL}

A crude sample of Polytran R (Ceca S. A., France) was purified by the method ${ }^{3,7}$ established for schizophyllan. The purified sample was dissolved in water at a concentration of about $1 \%$, and the solution was exposed to $19.5 \mathrm{kHz}$ sonic irradiation (Kaijo Denki, Model TA-6280N) for $70-100 \mathrm{~h}$ to fragment

* Present address: Research Laboratory, Taito Co., Higashishiriike-shinmachi, Kobe 653, Japan. 
the polymer into shorter chains. In this way, two samples (designated here as $\mathrm{L}$ and $\mathrm{P}$ ) were prepared. Each was divided into six or seven parts by fractional precipitation with water as the solvent and acetone as the precipitant. Two fractions, designated below as $\mathrm{L}-2$ and P-3, were chosen, reprecipitated from aqueous solutions into acetone, and freeze-dried from aqueous solutions. They were further dried in vacuo overnight before use. The viscosity-average molecular weights $M_{v}$ of samples L-2 and P-3 in water were $1.93 \times 10^{5}$ and $1.24 \times 10^{5}$, respectively.

Test solutions were prepared by mixing weighed amounts of a given sample and water in $5 \mathrm{~cm}^{3}$ stoppered flask. They were transferred into drum-shaped cells of $1 \mathrm{~mm}$ thickness and $1 \mathrm{~cm}$ diameter and examined under a Union Mec-3 polarizing microscope. The polymer concentration was expressed in terms of the weight fraction $w$ or the volume fraction $\phi$; the latter was calculated from $w$ with the specific volume of scleroglucan ${ }^{2}$ and the density of water.

ORD measurements were made on sample L-2, using a JASCO ORD/UV-5 recording spectropolarimeter. Preliminary experiments were performed on solutions with $w$ above 0.2 using rectangular cells of different thicknesses $d(20-350 \mu \mathrm{m})$ constructed according to Van et al. ${ }^{8}$ The results showed that, as in the case with the schizophyllan liquid crystal, ${ }^{8}$ the specific rotation $[\alpha]_{\lambda}$ at a fixed wavelength $\lambda$ was independent of $d$ and reproducible within $\pm 5 \%$ only when $d$ was smaller than about $100 \mu \mathrm{m}$. Thus, rectangular cells of $d \sim 80 \mu \mathrm{m}$ were used for solutions with $w$ above 0.15 . For solutions with $w$ below 0.15 , usual ORD cells with $0.5-10 \mathrm{~cm}$ thickness were used.

\section{RESULTS AND DISCUSSION}

\section{Microscopic Observation}

Aqueous solutions of sample L-2 at $25^{\circ} \mathrm{C}$ appeared dark between crossed polars throughout the cell when $w$ was lower than 0.14 . This

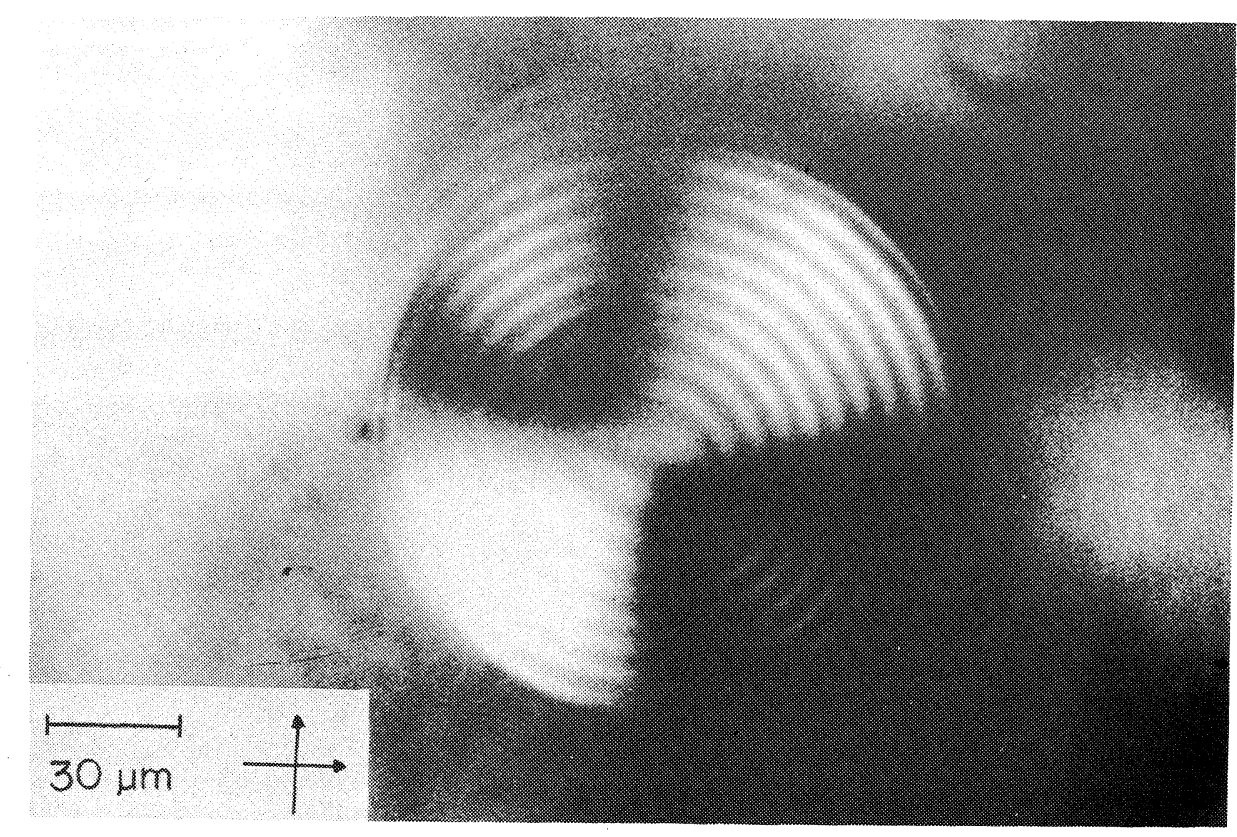

Figure 1. Spherulite in the aqueous solution of scleroglucan sample L-2 with $w=0.1567$ at $25^{\circ} \mathrm{C}$. The bright region on the top left-hand side shows the reflection of light from another large spherulite in the solution. 


\section{Liquid Crystal of Scleroglucan}

indicates that the solutions in this concentration region are isotropic. At $w$ slightly higher than 0.14 , birefringent phases with alternate bright and dark lines appeared as spherulites dispersed in a continuous isotropic phase a few $h$ after the solution had been placed in the cell. Figure 1 shows a photograph of a spherulite for the L-2 solution with $w=$
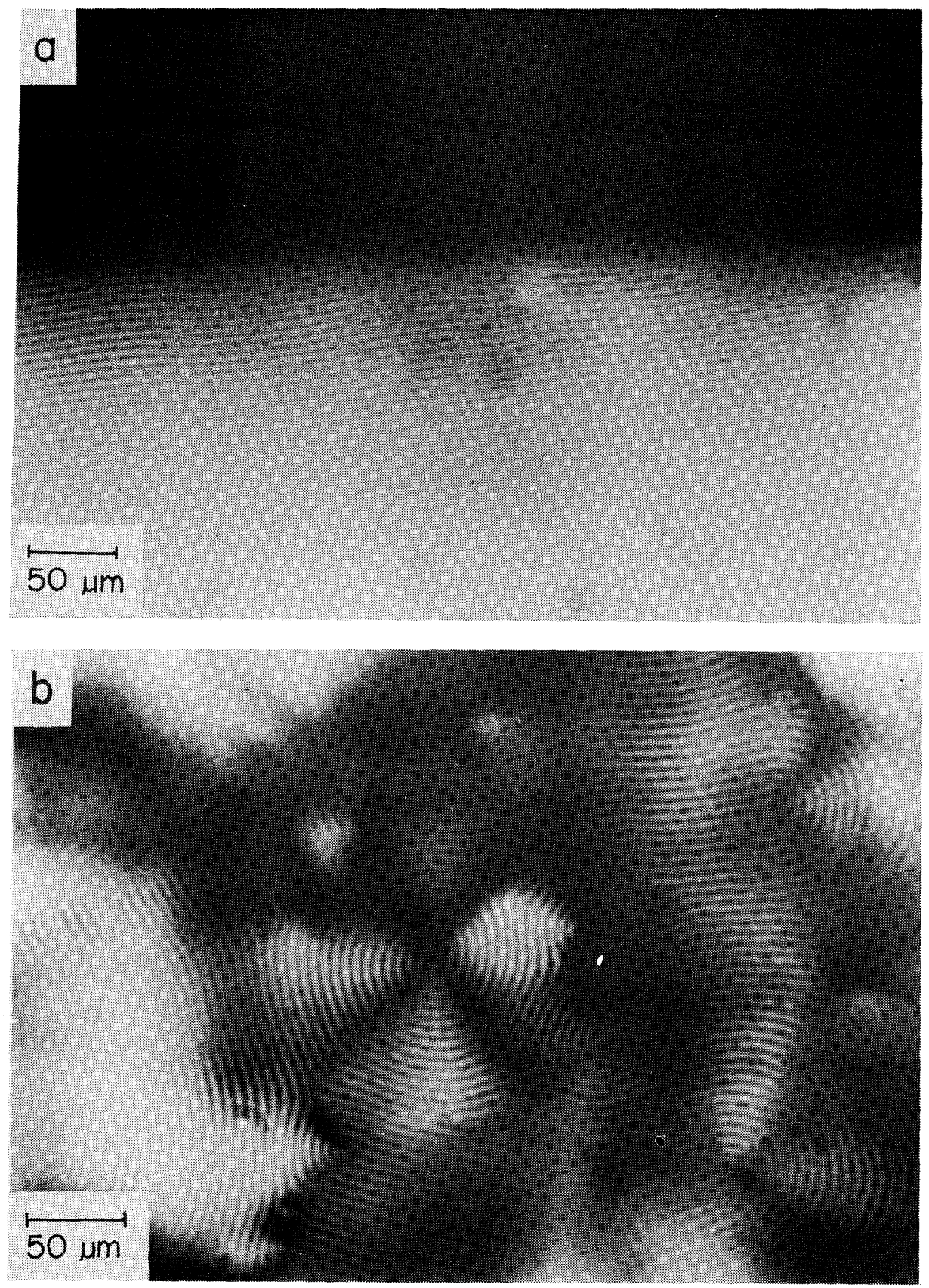

Figure 2. Microscopic patterns for the birefringent solution of sample L-2 with $w=0.2089$ at $25^{\circ} \mathrm{C}$. (a), near the cell wall; (b), near the center of the cell. 


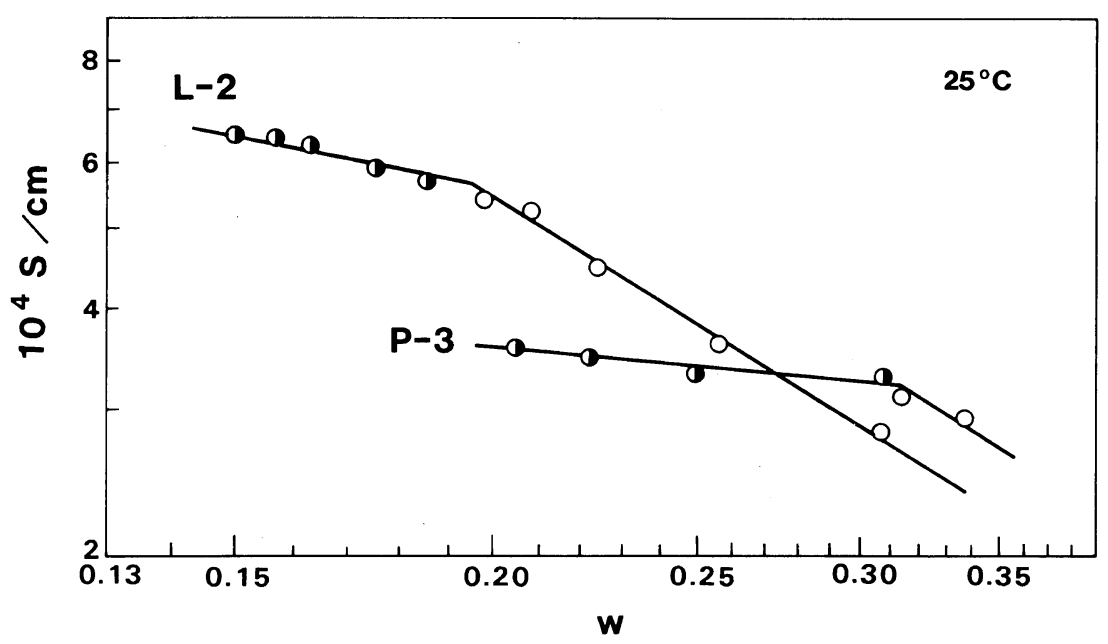

Figure 3. Double-logarithmic plots of $S$ vs. $w$ for samples L-2 and P-3 at $25^{\circ} \mathrm{C}$. ( ( ) ), biphasic solutions; (O), birefringent solutions.

\subsection{7 at $25^{\circ} \mathrm{C}$.}

When $w$ was raised to about 0.2 , the birefringent phase spread over the entire region of the solution and showed various colors. Figure 2 shows microscopic patterns for the birefringent solution of sample L-2 with $w=0.2089$ at $25^{\circ} \mathrm{C}$ near the wall [panel (a)] and center [panel (b)] of the cell. It can be seen that bright and dark lines in panel (a) run parallel to the cell wall, while those in panel (b) run in different directions to form fingerprint patterns. These patterns resemble those observed for the cholesteric liquid crystals of polypeptides $^{9-11}$ and polysaccharides. ${ }^{6,12,13}$

Similar observations were made at different temperatures between 25 and $20^{\circ} \mathrm{C}$ for samples L-2 and P-3, yielding results almost independent of temperature.

For a given biphasic or entirely birefringent solution, the distance $S$ between successive parallel lines was constant except near the cell wall. It was also found that $S$ remained unchanged at least for one month, though biphasic solutions tended to separate into isotropic and birefringent phases.

The measured values of $S$ for the two scleroglucan samples at $25^{\circ} \mathrm{C}$ are plotted doublelogarithmically against $w$ in Figure 3. Here, the half-filled and unfilled circles represent the data for biphasic and birefringent solutions, respectively. The data points for sample L-2 can be fitted by two straight liens intersecting at $w=0.194$. The line for $w \geq 0.194$ has a slope of -1.6 , which is fairly close to the reported slope of -1.9 for the schizophyllan liquid crystal. ${ }^{6}$ The indicated line for birefringent solutions of sample P-3 is drawn so as to have the identical slope of -1.6 . We note that when compared at the same $w, S$ for birefringent solutions of the two scleroglucan samples are about twice as large as those for schizophyl$\operatorname{lan}^{14}$ with comparable molecular weights.

\section{Optical Rotatory Dispersion}

Figure 4 displays ORD curves for solutions of sample L-2 with different $w$ at $21.5^{\circ} \mathrm{C}$. The curves for $w \geq 0.160$ are distinctly different from those for $w \leq 0.140$. The values of $[\alpha]_{436}$ and $[\alpha]_{589}$ at 436 and $589 \mathrm{~nm}$ read from these curves are plotted against $w$ in Figure 5. As $w$ increases, both $[\alpha]_{436}$ and $[\alpha]_{589}$ markedly increase in the region of $w$ between 0.14 and 0.19 and level off at constant values. Since the $w$ value of 0.14 almost coincides with the critical concentration (for sample L-2) at which spherulites begin to appear in the continuous iso- 
Liquid Crystal of Scleroglucan

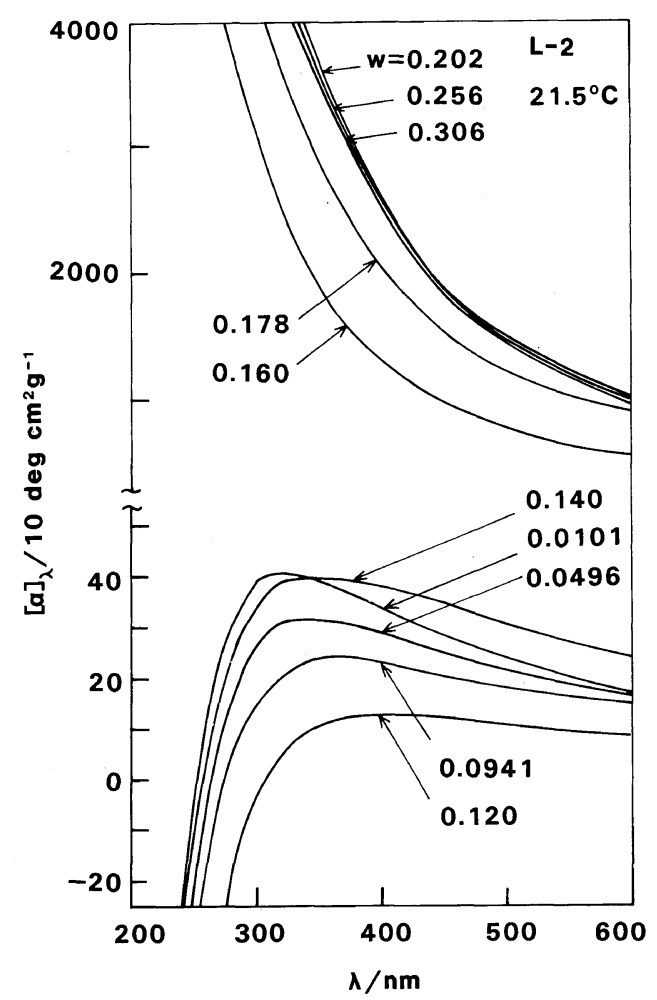

Figure 4. ORD curves for aqueous solutions of sample L-2 with different $w$ at $21.5^{\circ} \mathrm{C}$.

tropic phase, the sharp increases in $[\alpha]_{436}$ and $[\alpha]_{589}$ may be ascribed to the formation of birefringent phases. Furthermore, the leveling off values of $[\alpha]_{\lambda}$, about 200 and 100 $\operatorname{deg~}^{-1} \mathrm{~cm}^{2}$ for 436 and $546 \mathrm{~nm}$, respectively, are 700-500 times the infinite dilution values and comparable to the reported $[\alpha]_{436}$ and $[\alpha]_{589}$ for the cholesteric liquid crystal of schizophyllan. ${ }^{8}$ From these findings along with similarities of the observed microscopic patterns for scleroglucan to those for cholesteric liquid crystals, it may be concluded that the birefringent phase in aqueous scleroglucan is a cholesteric liquid crystal.

Figure 6 illustrates ORD data for the L-2 solution of $w=0.140$ at different temperatures. It can be seen that $[\alpha]_{\lambda}$ at a fixed $\lambda$ remarkably increases as the temperature is lowered from 21.5 to $15^{\circ} \mathrm{C}$. When the solution was examined by polarizing microscopy, birefringent phases were observed at 15 and $14^{\circ} \mathrm{C}$, but not at 21.5

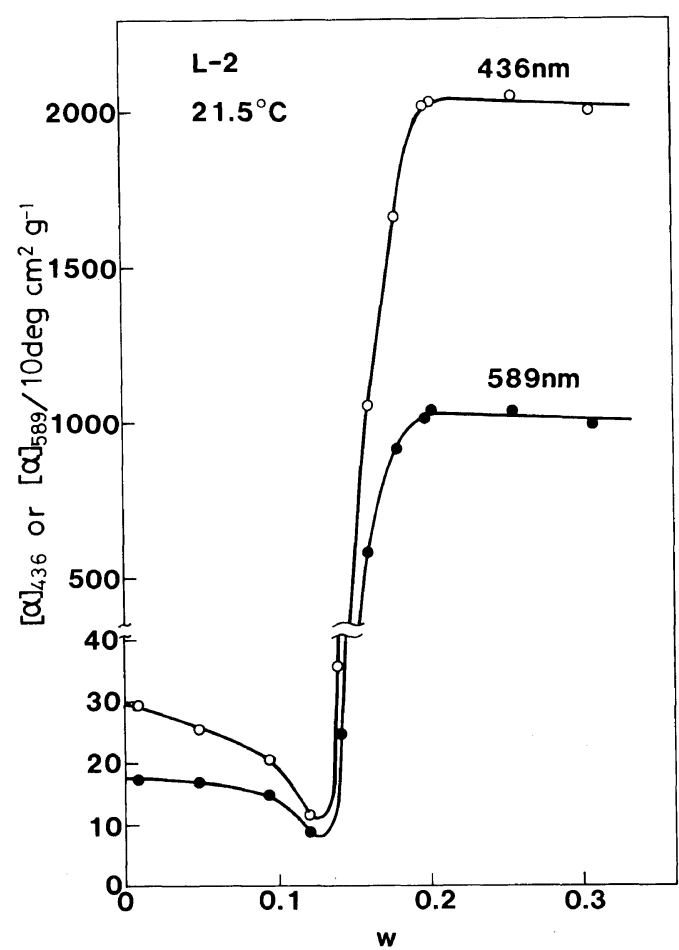

Figure 5. Concentration dependence of $[\alpha]_{436}$ and $[\alpha]_{589}$ for sample $\mathrm{L}-2$.

and $25^{\circ} \mathrm{C}$. These findings suggest that aqueous scleroglucan undergoes a thermally induced isotropic-liquid crystalline phase transition.

\section{Phase Diagram}

Our microscopic observations and ORD results indicate that the phase boundary between the isotropic and biphasic regions (the $\mathrm{A}$ point) and that between the biphasic and liquid crystalline regions (the $\mathrm{B}$ point) at room temperature are located at $w \sim 0.14$ and 0.19 for sample $\mathrm{L}-2$ and $w \sim 0.19$ and 0.31 for sample P-3. Referring to these $w$ values, we determined the temperature-concentration phase diagrams for the two scleroglucan samples by the methods described below.

\section{a. Microscopic Determination}

Solutions of sample L-2 with $w \sim 0.14$ and sample P-3 with $w \sim 0.19$ were examined between crossed polars, after being equilibrated at different temperatures $T$ of $11-33^{\circ} \mathrm{C}$. The 


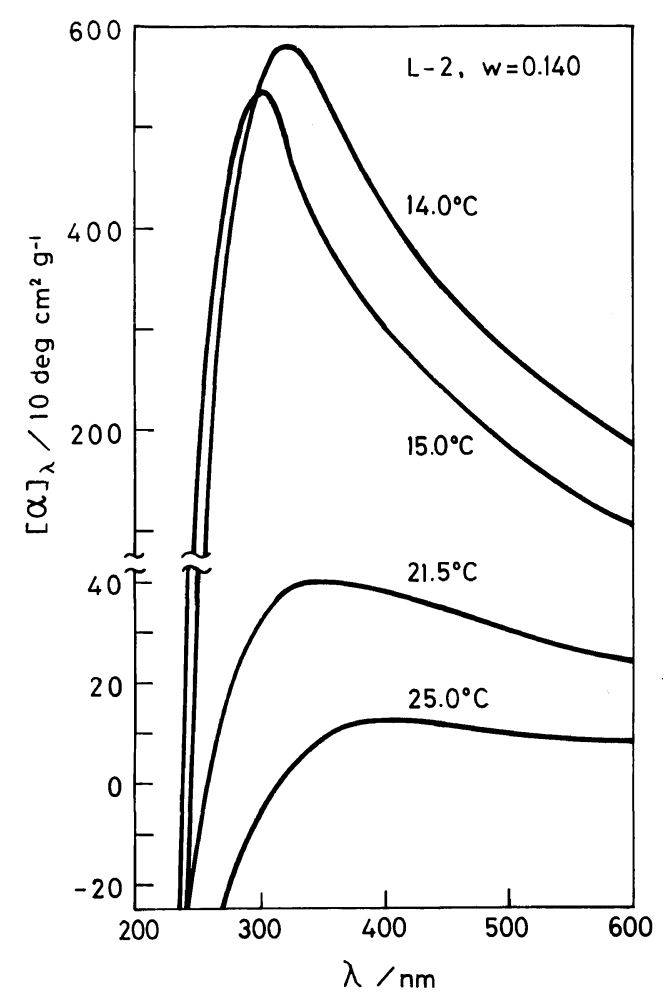

Figure 6. ORD curves for the solution of sample L-2 with $w=0.140$ at different temperatures. results are illustrated in Figure 7, in which the filled circles refer to isotropic solutions at given $T$ and $w$, and the unfilled circles to those that are biphasic. The line sandwiched between these filled and unfilled circles for each sample describes the A point. The B point concentrations estimated from the break points of the $S$ vs. $w$ curves in Figure 3 are also shown in Figure 7 by half-filled circles.

\section{b. Analysis of Biphasic Mixtures}

When a biphasic solution placed in a calibrated glass tube ${ }^{14}$ was spun at a rotor speed of $4000 \mathrm{rpm}$ (about 2000 gravities) for $1-2 \mathrm{~h}$ in a Hitachi $65 \mathrm{P}-7$ preparative ultracentrifuge, it separated completely into the isotropic and liquid crystalline phases. The volume fraction $\Phi$ of the isotropic phase and the molecular weight and weight fraction of the polymer in each phase were determined, as was done by Itou et $a l .{ }^{14}$; the redistribution of the polymer solute due to centrifugal force was considered negligible.

Figure 8 illustrates the results for sample L-2 at $25^{\circ} \mathrm{C}$. Here, the half-filled circles refer to the biphasic mixtures before separation, and the filled and unfilled circles to the separated isotropic and cholesteric phases, respectively. It

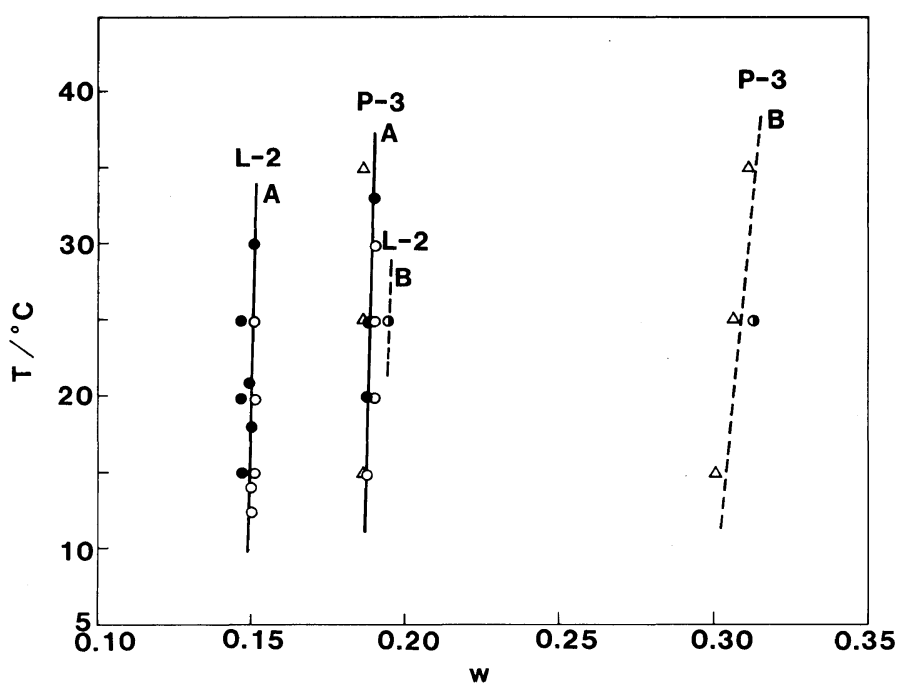

Figure 7. Phase diagrams for samples L-2 and P-3. Circles, from microscopic observations; triangles, from phase separation experiments. 

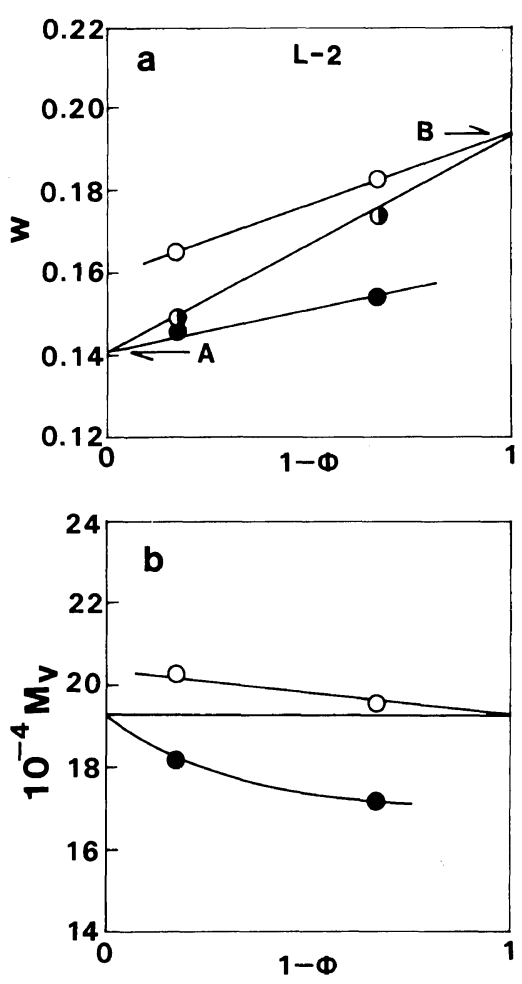

Figure 8. Phase separation data for sample L-2 at $25^{\circ} \mathrm{C}$. ( () , biphasic mixtures; $(\bigcirc)$ and $(\bigcirc)$, separated isotropic and cholesteric solutions, respectively. Arrows $\mathrm{A}$ and $\mathrm{B}$ indicate the $\mathrm{A}$ point and $\mathrm{B}$ point concentrations determined by polarizing microscopy.

can be seen in panel (a) that extrapolation of $w$ for the biphasic mixtures and separated phases to $\Phi=1$ and 0 yields phase boundary concentrations very close to the microscopically determined values (arrows A and B). In panel (b), $M_{v}$ in the liquid crystalline phase are larger than those in the isotropic phase, which indicates that sample L-2 undergoes molecular weight fractionation on phase separation.

The phase boundary $w$ estimated from similar data for sample P-3 are shown in Figure 7 by triangles. These $w$ agree with the microscopically determined values. Except for the B point concentrations for sample P-3, all the phase boundary concentrations for scleroglucan are close to the reported values for schizophyllan with comparable molecular weights. ${ }^{14}$

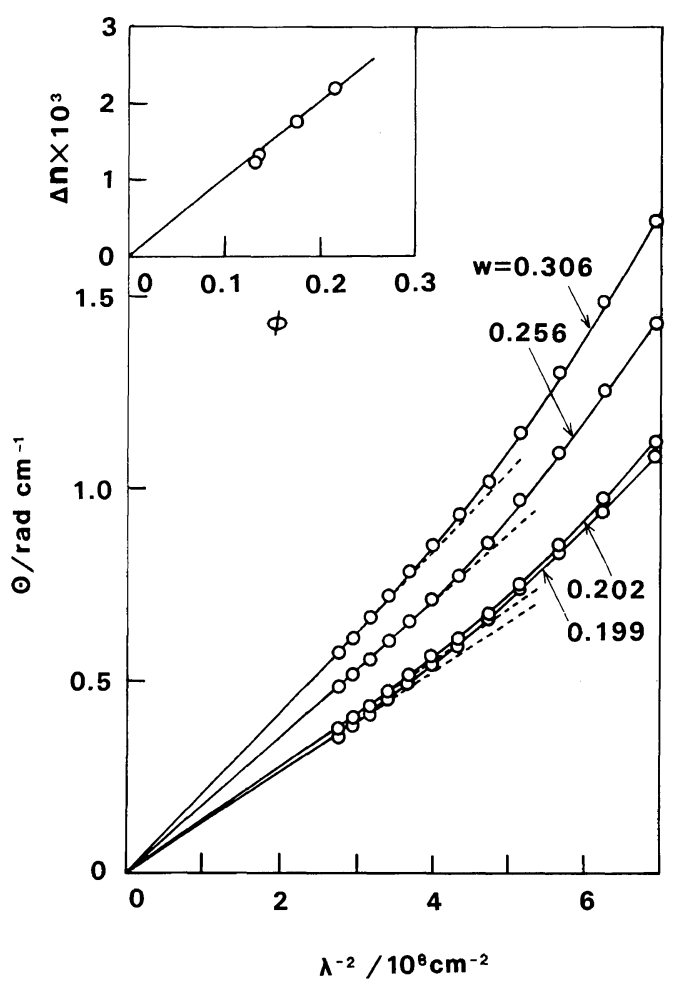

Figure 9. Plots of $\Theta v s . \lambda^{-2}$ for sample L-2. The insert shows the dependence of the layer birefringence $\Delta n$ on polymer volume fraction.

\section{Birefringence of the Cholesteric Layer}

When cholesteric solutions of sample L-2 in rectangular cells of $d \sim 80 \mu \mathrm{m}$ were examined microscopically between crossed polars, they appeared entirely uniform. This implies that in these cells, all the cholesteric planes are arranged essentially parallel to the cell surfaces. Thus, our ORD data for $w$ above the B point refer to the geometrical condition that the cholesteric planes are aligned in the direction normal to that of incident light, and may be analyzed by de Vries' theory ${ }^{15}$ for $\Theta$ (the optical rotation angle per unit length) of a cholesteric liquid crystal subjected to this condition.

The de Vries theory is written

$$
\Theta=\left(\pi / 4 \lambda^{2}\right)(\Delta n)^{2} P
$$

provided that $\Delta n P \ll \lambda \ll P$. Here, $P$ is the cho- 
lesteric pitch $(=2 S)$ and $\Delta n$ is the cholesteric layer birefringence defined by $\left(n_{\|}-n_{\perp}\right)$, with $n_{\|}$and $n_{\perp}$ being the refractive indices of a layer in the longitudinal and transverse directions of the director, respectively.

Figure 9 shows the plots of $\Theta$ vs. $\lambda^{-2}$ constructed from our ORD data for sample $\mathrm{L}$ 2 according to eq 1 . The curve fitting the plotted points for each $w$ follows the initial tangent indicated by a dashed line at smaller $\lambda^{-2}$, but tends to deviate upward in the region of $\lambda^{-2}$ above $3.5 \times 10^{8} \mathrm{~cm}^{-2}$. However, the deviation is not very pronounced below $\lambda^{-2}=$ $5 \times 10^{8} \mathrm{~cm}^{-2}$. Equating the initial slope of each curve to $\pi P(\Delta n)^{2} / 4$ and using the microscopically determined $2 S$ for $P$, we evaluated $|\Delta n|$ as a function of $w$.

The $|\Delta n|$ values so obtained were checked by retardation measurements (see ref 8 for the experimental details). It was found that, though not very accurate, the values of $\Delta n$ from retardation were positive and close to $|\Delta n|$ from $\Theta$. This agreement confirms the conclusion of Van et al. ${ }^{8}$ that eq 1 is valid for longer wavelengths.

The insert of Figure 9 shows that $\Delta n$ from $\Theta$ varies almost linearly with polymer volume fraction $\phi$. The indicated straight line yields a value of $1.0 \times 10^{-2}$ for $|\Delta n| / \phi$ which is close to $1.4 \times 10^{-2}$ reported for schizophyllan, ${ }^{8}$ but distinctly smaller than $4 \times 10^{-2}$ for xanthan, ${ }^{13}$ a $\beta$-1,4-D-glucan with ionic side chains. According to Van et al.,$^{8} \Delta n$ is related to the intrinsic birefringence $\left(n_{\alpha}-n_{\gamma}\right)$ of individual cylindrical macromolecules constituting a cholesteric layer by

$$
\Delta n=\left(n_{\alpha}-n_{\gamma}\right) \phi \xi(\phi)
$$

where $n_{\alpha}$ and $n_{\gamma}$ are the principal refractive indices in the longitudinal and transverse directions of the cylindrical molecule, respectively, and $\xi(\phi)$ is the order parameter characterizing the degree of ordering of molecules in the cholesteric solution. Thus, our $\Delta n / \phi$ value agrees with $\left(n_{\alpha}-n_{\gamma}\right)$ of the scleroglucan triple helix if $\xi(\phi)$ is unity in the range of $\phi$ studied.
At present, however, no information is available on $\xi(\phi)$ for cholesteric liquid crystals.

Doi $^{16}$ theoretically predicted that $\xi(\phi)$ for a nematic liquid crystal monotonically increases from 0.5 and approaches unity as $\phi$ increases from the value at the B point toward unity. If Doi's $\xi(\phi)$ is applied to our $\Delta n$ data, values of $\left(n_{\alpha}-n_{\gamma}\right)$ for scleroglucan decrease from $1.7 \times 10^{-2}$ to $1.3 \times 10^{-2}$ with increasing $\phi$. Taken literally, this implies that $\xi(\phi)$ for scleroglucan is less dependent on $\phi$ than the theoretical prediction by Doi.

However, we cannot claim too much accuracy in the numerical results obtained, because Doi's theory is concerned with nematic liquid crystals and de Vries' theory can describe experimental data only in the limited wavelength range. Thus, it is probably safe to conclude that the intrinsic birefringence of the scleroglucan triple helix is about $1.5 \times 10^{-2}$ and indistinguishable from that of the schizophyllan triple helix.

\section{CONCLUDING REMARKS}

We have found that the cholesteric liquid crystal of aqueous scleroglucan resembles that of aqueous schizophyllan in thermodynamic and optical properties. This is consistent with the previous finding ${ }^{1,2}$ that these two glucans are chemically indistinguishable and have essentially the same triple helical structures.

However, there is a distinct difference in $S$ (hence in $P$ ) between the two glucans: $P$ for scleroglucan is almost twice as large as that for schizophyllan when compared at the same $w$. A cholesteric liquid crystal may be treated as a pile of thin layers, ${ }^{17}$ in which successive layers are twisted by a small angle $\beta$. Therefore, the above difference in $P$ implies that the two liquid crystals differ either in the distance between successive layers, in $\beta$, or in both. If the inter-layer distance for the two glucans are the same, $\beta$ for scleroglucan should be roughly half that for schizophyllan. In any case, the two liquid crystals must be different in inter- 
layer interaction. This may be related to the solubility difference between the two glucans found in the previous dilute-solution study. ${ }^{2}$

As can be seen in Figure 9, our ORD data follow de Vries' prediction only in a limited range of $\lambda^{-2}$. We have found within the wavelength range examined, the ORD curve for any of our cholesteric solution is described accurately by the Drude equation. This is not specific to the scleroglucan liquid crystal but can be seen also for the liquid crystals of schizophyllan $^{8}$ and poly $(\gamma$-benzyl L-glutamate). ${ }^{18}$ Further theoretical study is needed for clarification of this characteristic ORD behavior.

\section{REFERENCES}

1. T. Yanaki, T. Kojima, and T. Norisuye, Polym. J., 13, 1135 (1981).

2. T. Yanaki and T. Norisuye, Polym. J., 15, 389 (1983).

3. T. Norisuye, T. Yanaki, and H. Fujita, J. Polym. Sci., Polym. Phys. Ed., 18, 547 (1980).
4. T. Yanaki, T. Norisuye, and H. Fujita, Macromolecules, 13, 1462 (1980).

5. Y. Kashiwagi, T. Norisuye, and H. Fujita, Macromolecules, 14, 1220 (1981).

6. K. Van, T. Norisuye, and A. Teramoto, Mol. Cryst. Liq. Cryst., 78, 123 (1981).

7. K. Tabata, W. Ito, T. Kojima, S. Kawabata, and A. Misaki, Carbohydr. Res., 89, 121 (1981).

8. K. Van, T. Asakawa, and A. Teramoto, Polym. J., 16, 61 (1984).

9. C. Robinson, Trans. Faraday Soc., 52, 571 (1956).

10. W. G. Miller, Ann. Rev. Phys. Chem., 29, 519 (1978).

11. K. Ito, T. Kajiyama, and M. Takayanagi, Polym. J., 12, 305 (1980).

12. J. Bheda, J. E. Fellers, and J. L. White, Colloid Polym. Sci., 258, 1335 (1980).

13. G. Maret, M. Milas, and M. Rinaudo, Polym. Bull., 4, 305 (1980).

14. T. Itou, K. Van, and A. Teramoto, J. Appl. Polym. Sci., in press.

15. H. de Vries, Acta Cryst., 4, 219 (1951).

16. M. Doi, J. Polym. Sci., Polym. Phys. Ed., 19, 229 (1981).

17. S. Chandrasekhar, "Liquid Crystals," Cambridge Univ. Press, Cambridge, 1977, Chapter 4.

18. D. L. Patel and D. B. DuPré, J. Chem. Phys., 72, 2515 (1980). 